\title{
Companion: A Social Support Generating Application for Lonely and Socially Isolated Individuals using Lazy Supervised Algorithm
}

\author{
Marisa M. Buctuanon, Xavier Comabig and Wavina Vivienne Mayola \\ University of San Jose-Recoletos \\ marisamahilum@gmail.com
}

\section{Abstract}

The adverse effect of depression, which is a significant health problem associated with loneliness and social isolation, leads to suicidal ideation, alcohol use, cardiovascular disease, and altered brain function. Administering them with appropriate and effective mental health outreach and treatment services can prevent and alleviate these effects. Studies state that being with individuals who can help them cope with their situation under the supervision of a professional psychologist or psychiatrist can help them achieve a state of well-being. Thus, this study develops Companion, an app that utilizes the KNN algorithm to create a social support group that matches a seeker to the group based on his interest and problem at hand. He finds new friends with whom he can feel a sense of care, love, understanding, and belongingness. It comes with a set of physical activities designed for the group. The algorithm yields an $84 \%$ accuracy rate after evaluating the result.

Keywords: social support group, loneliness, social isolation, KNN, instance-based algorithm, a lazy supervised algorithm

\subsection{Introduction}

Social support means having a network of family and friends whom an individual can turn to when facing a personal crisis (Cherry, 2018). Immediate assistance for individuals who want to spend time with people who care about them is necessary. The subjective feeling of being alone, separated, or apart from others is referred to as loneliness (Cattan, White, Bond, \& Learmouth, 2005) (Wenger, Davies, Shahtahmasebi, \& Scott, 1996) (Tomaka, Thompson, \& Palacios, 2006) which often leads to a significant health problem called depression. According to (Garlow et al., 2008), an individual with higher depression has more tendencies to think of suicide. Since there is a strong relationship between the severity of depressive symptoms and suicidal ideation, it is therefore, essential to provide adequate mental health outreach and treatment services. The care, love, and value that an individual receives from a network of mutual obligations (Cobb, 1976), lead to a decreased risk of depression, suicide, alcohol use, cardiovascular disease, and altered brain function (Cherry, 2018). 
Social isolation is the physical separation from other people such as living alone or staying away from a specific companion (Cattan, White, Bond, \& Learmouth, 2005) (Wenger, Davies, Shahtahmasebi, \& Scott, 1996) (Tomaka, Thompson, \& Palacios, 2006). The situation induces behavioral and neuroendocrine responses relevant to depression (Grippo et al., 2007). Both loneliness and social isolation are related to adverse health outcomes, which could be naturally suppressed by social support since it is associated with positive health outcomes (Tomaka, Thompson, \& Palacios, 2006). These studies convey that having a social support group influences the well-being of an individual to cope with a personal crises or stressful events, which could turn to healthy being.

In the absence of a tangible human companion, mobile applications provides the opportunity to access several social support applications available to the public. Social media applications nowadays allow everyone to share posts, chat with friends, comment on a post, and do other social interaction activities. The site (Top 25 Mental Health Apps for 2018: An Alternative to Therapy, n.d.) suggests the top 25 mental health applications that can help an individual handle his need for emotional, psychological, and social wellbeing. However, this study will focus mainly on the applications related to social support mentioned by the site. For instance, Happify has scientifically prepared engaging games and activities to help the user focus on positive thoughts (Happify: ScienceBased Activities and Games, n.d.). UCSF PRIME has a social network interface by means of which, users can interact with peers, have motivational coaches, and goal-focused challenges (UCSF PRIME, n.d.). Stigma is an application with a social network feature that allows users to connect with peers and share their journal to get support from others
(Stigma: Mood Tracker and Journal, n.d.). Another one is Talkspace Online Therapy (Talkspace, n.d.), through which an individual can talk to trained professionals whenever he needs professional intervention.

Allowing other users of any application to give feedback or send a message to a person who needs social support without predetermining if they are capable of doing so can worsen the situation. The social media applications mentioned above, including Stigma and UCSF Prime, allow users to perform social activities online. If not controlled or managed correctly, the person who needs social support might be in danger, considering that there could be unnecessary or unsolicited comments or messages that could worsen the situation. Talking to someone online might not be enough since social support is a multidimensional (Tomaka, Thompson, \& Palacios, 2006). Tangible support, a type of social support, which demands physical assistance, is missing in Talkspace Online Therapy and UCSF PRIME. This type of social support might be necessary to uplift the current situation of an individual. Though the games in Happify and UCSF PRIME are prepared by psychiatrists or psychologists, the unavailability of social interaction in these games, does not give them the help they want from a group of individuals.

To address the different gaps in the social support apps mentioned above, the researchers developed the application known as Companion. Companion has mobile and web components. In the mobile part, two types of users exist, a seeker and a facilitator. A seeker is the one who needs social support. A facilitator is the one who is a licensed psychiatrist or psychologist who provides the social support needed by the seeker. The ultimate goal of this application is to create a social support 
group with seekers who have similar profiles and problems at hand. The facilitator will conduct counseling sessions through the application and will assist the seekers in performing the system generated physical activities. The lazy supervised algorithm called K-nearest neighbor (KNN) creates the social group of seekers and determines the facilitator of the group. The algorithm also recommends a set of activities to the facilitator. Online social interactions are also featured in the application such as personal chitchat to the facilitators, posting to a freedom wall, reading inspirational quotes, and watching motivational videos. The facilitator will only see the post in the freedom wall to secure the seeker's privacy from other seekers, and to avoid other seekers to feel the same way. The system preselects the quotes and videos with the use of the administrative application.

\section{Review of Related Literature}

According to (Cutrona \& Russell, 1993), social support, and a sound psychological and physical health among diverse groups of individuals facing a variety of physical or mentally demanding situations are highly related to each other. It supports the idea that "Friends can be good medicine!" Creating a group of individuals implies that having friends is an excellent way to help them in facing their situations. Additionally, it is not just social support that is essential to our health, but also social integration (Cherry, 2018). Social integration is the actual participation of an individual that involves emotions, intimacy, and a sense of belongingness. Although (Cohen \& Wills, 1985) states that social integration and interpersonal relationship or functional support are not directly correlate to each other, this study would like to explore the possibility of combining social support, and social integration in this application.

The study of Gazda (1969) found out that group counseling is essential, not just for prevention, but also for remediation of problems that relate to poor coping behaviors at certain developmental stages. It is crucial to select counselors and group members to promote group cohesiveness in building positive feelings or expectancies toward the group. There should be a goal to achieve to give the group a sense of purpose and direction; for this reason, this study commissions the licensed psychiatrist or psychologist to do group counseling and find the best group of seekers who have similar interests and problems at hand.

(Stults-Kolehmainen \& Sinha, 2014) suggest that physical activity promotes positive changes in one's mental health and ability to cope with stressful events. It lessens the rates of depression, negative affectivity, and anxiety. Since taking a leisure trip provides opportunities for relaxation, a weekend getaway can help people recover from stress (Chen, Petrick, \& Shahvali, 2016). Thus, meeting with a group of individuals in a convenient and conducive venue where seekers can perform physical activities is factored in this study.

Finding the right algorithm to group seekers is significant. Social group optimization (SGO) is a population-based optimization technique that looks for the best person to disseminate knowledge among the entire members of the group to solve the group problem (Satapathy \& Naik, 2016). However, in this study, SGO is not implemented since the person who has the best knowledge in helping the group to solve their problem is a licensed psychiatrist or psychologist. In this study, K-nearest neighbor (KNN) is used to find the best group of seekers and facilitators. Nearest Neighbor Classification is so simple that it classifies a specific 
class based on identifying its nearest neighbors (Kataria \& Singh, 2013). KNN is a conventional nonparametric classifier that provides excellent performance for optimal values of $k$. It is a type of instance-based or lazy supervised algorithm since it does not build any model and does its work at prediction time (Andrade, 2016). The computation is deferred until classification takes place. Once classified, it discards the intermediate results and will make the full process starts again (Aha, 1997). This algorithm is suitable when the labels are not initially available and collected at that instant. It employs distance or proximity metrics to determine which neighbors are near using Euclidean distance. Although there are other metrics for KNN, Euclidean distance is known to be easy, efficient, and productive (Kataria \& Singh, 2013).

\subsection{Methodology}

The entire system has two different frameworks, lonic and Laravel. Ionic is for the mobile appcation and Laravel is for the web application and web services. Application programming interfaces (APIs) such as Google Maps (Maps JavaScript API, n.d.), Geolocation (Geolocation API, n.d.), Forismatic Quote (Quotes and expressions. The most inspiring expressions of mankind., n.d.), and Youtube API (Youtube API, n.d.) retrieve the user's location, inspirational quotes, and videos, respectively. The Google Maps and Geolocation APIs utilize only the location of the user. These APIs do not require other credentials, such as names, interests, and problems.

\section{Grouping Users for Talk Circle}

A talk circle is the term used to the generated social support group of the system. A talk circle is composed of one facilitator and four seekers. This number of seekers ensures that the facilitator can manage the group. At the start, the seeker already identified the problems at hand, interests, and available time and day. The facilitator sets his specialization and possible time and day at which he can meet the group. Both processes are carried out during the registration phase using the mobile application.

Since KNN is an instance-based or lazy supervised algorithm, there is no training time or learning phase for the classification. The classification process occurs every time there is a request to classify; for this reason, this kind of algorithm does not learn from the training set, but does more computation on the test set. Here, the system needs to classify the unlabeled seeker by searching for the nearest facilitator in the entire training set. The training set is composed of the specializations attributed to the facilitators who are available to facilitate the talk circle.

The initial activity in the first phase of KNN is to load the data from the database. In this phase, the system will check if there is a facilitator available for group counseling. With the availability of a facilitator, all the seekers who are looking for advice will be retrieved. The second phase is to initialize $\mathrm{K} . \mathrm{K}$ here refers to the number of nearest neighbors. In this case, the $K$ is four, which represents the number of seekers who will be the nearest neighbors of the facilitator. Prior to calculating the distance from the facilitator to each seeker, the system needs to prune this data set.

This process of pruning makes sure that the group of seekers matches the available time and day of the facilitator. The system will check if there are enough seekers that match the facilitator's possible time and day(s) for a particular week. Table 1 shows the different cases if a facilitator's $(F)$ 
possible time and seeker's (S) possible time have met specific criteria to be part of the talk circle.

Table 1. Acceptable Cases for Matching Two Time Frames

\begin{tabular}{cc}
\hline Time Start & Time End \\
\hline $\mathrm{S}=\mathrm{F}$ & $\mathrm{S}=\mathrm{F}$ \\
(e.g. 9:00 AM=9:00 AM) & (e.g. 12:00 PM=12:00 PM) \\
\hline $\mathrm{S}=\mathrm{F}$ & $\mathrm{S}>\mathrm{F}$ \\
(e.g. 9:00 AM=9:00 AM) & (e.g. 1:00 PM $>12: 00 \mathrm{PM})$ \\
\hline $\mathrm{S}<\mathrm{F}$ & $\mathrm{S}>\mathrm{F}$ \\
(e.g. 8:00 AM<9:00 AM) & (e.g. 1:00 PM $>12: 00 \mathrm{PM})$ \\
\hline
\end{tabular}

The different time frame cases shown in Table 1 indicate that the system makes sure that the meeting time is within the facilitator's available time. For example, using the third case time frame, the seeker's start time is earlier than the facilitator, and the facilitator end time is before the seeker's end time. For instance, if the seeker's available time starts at 8:00 AM and ends at 1:00 PM, it is matched with a facilitator who can accommodate a seeker from 9:00 AM until 12:00 PM. Since the system maximizes the availability of the facilitator, it does not consider other cases such as cases displayed in Table 2. For instance, if the facilitator is available at 8:00 AM, he will not be matched with a seeker who will be available later than the set time.

Table 2. Sample Invalid Cases for Matching

\begin{tabular}{cc}
\hline Time Start & Time End \\
\hline $\mathrm{S}>\mathrm{F}$ & $\mathrm{S}=\mathrm{F}$ \\
(e.g. 9:00 AM>8:00 AM) & (e.g. 12:00 $\mathrm{PM}=12: 00 \mathrm{PM})$ \\
\hline $\mathrm{S}>\mathrm{F}$ & $\mathrm{S}>\mathrm{F}$ \\
(e.g. 9:00 AM $>8: 00 \mathrm{AM})$ & (e.g. 1:00 PM $=12: 00 \mathrm{PM})$ \\
\hline
\end{tabular}

The process of calculating the distance from the facilitator to each seeker who passed the pruning process begins in this phase. The system checks if the seekers are within five kilometers away from the facilitator to get the nearest facilitator to the seeker. Using the APIs of Google Maps and Geolocations, the system uses Equation 1 with the addresses of the facilitator and the seeker. Equation 1 (Movable Type Scripts, n.d.) is commonly used by Google Maps to compute the shortest distance since this formula is suitable for distance computation on a sphere, given their longitudes and latitudes.

\section{Equation 1: Haversine Distance Formula}

$$
\begin{array}{ll}
\text { Haversine } & a=\sin ^{2}\left(\frac{\Delta \phi}{2}\right)+\cos \phi_{1} \cdot \cos \phi_{2} \cdot \sin ^{2}\left(\frac{\Delta \lambda}{2}\right) \\
\text { formula: } & c=2 \cdot \operatorname{atan} 2(\sqrt{a}, \sqrt{(1-a)}) \\
& d=R \cdot c
\end{array}
$$

Where $\phi$ is latitude, $\lambda$ is longitude, R is earth's radius (mean radius $=6,371 \mathrm{~km}$ ); note that angles need to be radians to pass trig functions

The system evaluates whether those who passed the five-kilometer radius, match the specialization of the facilitator. Table 3 shows all the categories of specialization and the related problems that can be handled by the facilitator. Additionally, the information in Table 3 comes from the guidance office of a university.

Table 3. Facilitator Specialization and Related Problems

\begin{tabular}{ll}
\hline \multicolumn{1}{c}{ Specialization } & Related Problems \\
\hline Addiction and & Health Problem \\
Substance Abuse & Alcohol and Drug-related \\
& Problem \\
\hline Career and & Work and Career Problem \\
Vocational & Financial Problem \\
\hline
\end{tabular}


Continuation of Table 3 (Facilitator Specialization and Related Problems)

\begin{tabular}{ll}
\hline \multicolumn{1}{c}{ Specialization } & Related Problems \\
Domestic & Social and Relationship Problem \\
& Personal Issues \\
& Violence Problem \\
& Family Problem \\
\hline Grief & Loss of Loved One \\
& Social and Relationship Problem \\
& Personal Issues \\
& Family Problem \\
\hline LGBT & Social and Relationship Problem \\
& Personal Issues \\
& Gender Problem \\
& Family Problem \\
\hline Marriage and & Social and Relationship Problem \\
Family & Financial Problem \\
& Family Problem \\
\hline Mental Health & Health Problem \\
& Social and Relationship Problem \\
& Personal Issues \\
\hline Wcademic or & Work and Career Problem \\
& Academic Problem \\
& Financial Problem \\
\hline & \\
&
\end{tabular}

The figures shown in Table 4 are the decimal representations of the range selected by the seeker at the very start of the registration. The mapping of seekers is done by summarizing their problems and getting the average number of issues for every specialization. Since every specialty has a different issue, the scores of each issue will be summarized to determine the rating of the specialty. For example, if the seeker has gender and family problems, the ratings of these problems are reflected in the LGBT specialization as average.
Table 4 shows the average sample computation of each seeker per specialization. The score ranges from zero to one. Zero means no problem at all, and one means that the seeker needs much help in that area. It is the seeker who determines the score for each issue. For example, Jules has issues on the financial and family under Marriage and Family specialization. He scored them 0.9 and 0.5 , respectively. After computing for their average, the Marriage and Family specialization will have a score of 0.7.

Table 5 shows the list of interests that the seeker can choose from the application. Starting from 11, the areas are arts, crafts, literature, music, gaming, movies, puzzles, sports, adventure, and get-together. The seeker gives these scores during the registration process. The values are represented by flags that represent zero as not interested, and one as interested. The names in Table 4 and Table 5 are artificial data to observe data privacy. The scores in both tables solve the distances between facilitator and seekers. The system uses Equation 2 (Euclidean Distance, n.d.) for this computation. This process is different from the previous computation that uses Equation 1 with the latitude and longitude of the seeker and the facilitator. Equation 2 is used by the system to evaluate the data of the nearest seekers that can be accommodated by the facilitator based on their problem. KNN employs both computations. However, in the previous process, Equation 1 was used instead of Equation 2 since the earth is spherical. Equation 2 is applicable only for the distance between straight lines.

Equation 2: Euclidean Distance Formula

$$
\sqrt{\sum_{i=1}^{k}\left(x_{i}-y_{i}\right)^{2}}
$$


Table 4. Average Score of Specialization per Seeker

\begin{tabular}{lcccccccc}
\hline Seeker & Addiction & Career & Domestic & Grief & LGBT & Marriage & Mental & Academic \\
\hline Jules & 0 & 0 & 0.5 & 0.5 & 0.6 & 0.7 & 0.3 & 0 \\
Andrea & 0.5 & 0.5 & 0.25 & 0.25 & 0.2 & 0.3 & 0.3 & 0.6 \\
Rhaylyn & 0.5 & 0.5 & 0.5 & 0.5 & 0.4 & 0.7 & 0.7 & 0.3 \\
Eric & 0.5 & 0 & 0 & 0.25 & 0 & 0 & 0 & 0.3 \\
Sean & 0 & 0.5 & 0.25 & 0.25 & 0.2 & 0.3 & 0.3 & 0.7 \\
\hline
\end{tabular}

Table 5. Interests per Seeker

\begin{tabular}{lcccccccccc}
\hline Seeker & $\mathbf{1 1}$ & $\mathbf{1 2}$ & $\mathbf{1 3}$ & $\mathbf{1 4}$ & $\mathbf{1 5}$ & $\mathbf{1 6}$ & $\mathbf{1 7}$ & $\mathbf{1 8}$ & $\mathbf{1 9}$ & $\mathbf{1 1 0}$ \\
\hline Jules & 0 & 0 & 1 & 1 & 1 & 1 & 1 & 0 & 1 & 0 \\
Andrea & 1 & 1 & 0 & 1 & 1 & 1 & 1 & 1 & 1 & 1 \\
Rhaylyn & 1 & 0 & 1 & 1 & 1 & 0 & 0 & 0 & 0 & 0 \\
Eric & 0 & 0 & 0 & 1 & 0 & 0 & 0 & 0 & 0 & 1 \\
Sean & 0 & 1 & 1 & 1 & 1 & 0 & 1 & 1 & 1 & 1 \\
\hline
\end{tabular}

After this, the next phase of KNN is to sort the seekers based on the computed distance, from the shortest distance to the longest. Then, in the next phase, the system picks the top four seekers. And lastly, they will be labeled as part of the facilitator's group.

The facilitator will become the fifth member of the talk circle. The system stores this information in the database. The talk circle membership of the seeker changes every time the seeker looks for a social support group. If for some reason the seeker would not want to continue joining the group, the seeker can cancel the talk circle. However, the talk circle will remain for the rest of the seekers.

\section{Recommendation for Activities}

The next phase after generating the talk circle is the recommendation of activities per talk circle. In this phase, the system will get the group's general problems and interests and sorts out the activities for the facilitator. Using the KNN algorithm that utilizes Equation 2, the system cross- checks the group's problems and interests with the activities' target problem, and recommends the result to the facilitator. Based on research, the activities present in the database present evidence that they are suitable for interventions. These activities are added through the web application by the administrator. The system administrator manages the addition and deletion of activities in the database. All activities will be sorted based on their scores in descending order, thus allowing the facilitator to see the highest rated first.

\section{Recommendation of Nearby Venues}

All venues found in Google Places suitable for group activities will be retrieved based on the members' location. Using its API, the researchers set the category of the place as a park, tourist attraction, and other sites that have a bucolic or pastoral scenery and saves the results to the database. These types of sites provide relaxation to the seekers. Equation 3 (Equation of a Circle, 2007) locates the group's average position, and thus the 
center for the geofencing.

Equation 3: Center Point Equation

$$
(\mathrm{x}-\mathrm{h})^{2}+(\mathrm{y}-\mathrm{k})^{2}=\mathrm{r}^{2}
$$

After getting the center point, using Equation 1 , the system will check whether the venue is within the geofence radius. The location of the farthest member of the group will be the maximum radius for the geofencing. The system recommends to the facilitator the geofence result of a specific venue, which is within the area of all the members of the talk circle.

Table 6. Sample Venues from Google Places

\begin{tabular}{lc}
\hline Venue & $\begin{array}{c}\text { Distance from the } \\
\text { Center Point via Car }\end{array}$ \\
\hline $\begin{array}{l}\text { Sirao Flower Garden, } \\
\text { Cebu City }\end{array}$ & $21 \mathrm{~km}$ \\
$\begin{array}{l}\text { Baguio De Cebu } \\
\text { Eco-Mountain Park } \\
\text { Adventure, Balamban, } \\
\text { Cebu }\end{array}$ & $16.6 \mathrm{~km}$ \\
$\begin{array}{l}\text { Osmeña Peak, Badian, } \\
\text { Cebu }\end{array}$ & $146 \mathrm{~km}$ \\
\hline
\end{tabular}

Table 6 shows an example of the retrieved venues using Google Places API. Supposing, the center point of the group is $10.382170,123.782190$, the latitude and longitude, respectively. Locating this through the Google Map, this is Sudlon I, Cebu City. The farthest member is living in Minglanilla, Cebu, which is $42.4 \mathrm{~km}$ away from the center point via car. Looking at the result in Table 6, only Sirao Flower Garden and Baguio De Cebu Eco-Mountain
Park Adventure will be recommended since they don't exceed the $42.4 \mathrm{~km}$ distance.

\section{Social Media Activities}

The social media activities, such as the posting of status on the freedom wall is saved in the server together with the selected feeling of the seeker. Once the seeker posts his status and opinion, the system identifies the top three categories with which his post is associated. The list of categories is the specialization shown in Table 3. Through the use of Forismatic Quote API, the system retrieves the quotes that motivate the seekers to uplift their mood or feeling. The retrieval of quotes from the API is done before the seeker posts his status and opinion; therefore, the quotes are pre-classified by the system administrator. The system evaluates the content of the opinion by counting the number of similar words of the post to each keyword in the database. Each quote has a set of keywords stored by the system administrator. The seeker sees the top three highest similarity count quotes. If there is no matching quote or if the number of matching quotes is less than three, the system randomly retrieves the lacking quotes from the database.

With the use of YouTube API, the system fetches videos that inspire the seekers to think positively and feel good. The server stores the links of these videos. The system administrator sets the classification of the videos and equates them with a set of keywords. The system retrieves the top 15 videos based on the scores acquired during the counting of similar words of the post to the keywords of each video. The videos are displayed in descending order to the seeker. As with the quotes, the system retrieves the lacking videos randomly 
if there are no or fewer videos that correspond to the post. Moreover, the randomness of the retrieval ensures that the quotes and videos have an equal chance to be displayed to the seeker.

\subsection{Results and Discussion User Interface}

This section shows the different user interfaces (UIs) of the mobile application. The seeker who wants to use the application needs to register before entering his credentials, as shown in Figure 1. Upon registration, the seeker selects his interests and sets his problem at hand. Securing the privacy of the seeker is very crucial. Only the facilitators who are licensed psychologists or psychiatrists can see the problem and interact with the seeker. The system administrator scrutinizes the registration of the facilitator. The administrator checks the credentials and certificates uploaded by the facilitator. It is the right of the administrator to accept, decline, and even revoke the participation of the facilitator. Once the seeker and the facilitator finishes the registration process, they can now login using Figure 1. In cases that they forget their login credentials, they can click the forgot password in Figure 1.

Figure 2 displays the quotes that can relieve or lessen the negative feeling of the seeker. Figure 3 is a screenshot of a freedom wall where the seekers can express their feelings by writing and adding emoticons to their posts. The facilitators who want to reach out and counsel the seeker can respond to the post. Again, only the facilitators can respond or comment on the post to mitigate the chance of unnecessary comments from other seekers.
The seeker can access not only the quotes but also the motivational videos every time he creates a post. Figure 4 shows sample videos retrieved from the server. Furthermore, every time the seeker clicks the talk circle button, the system cues the seeker's details in the database and eventually assigns a facilitator and his co-members that match his interest and problem at hand. The system does not instantly generate the group for the seeker. The system still needs to check whether there is an available facilitator or not. Since the system does the grouping, the facilitator doesn't know the seeker's details beforehand. Once done, the seeker sees Figure 5. The location, time, and date are already approved by the facilitator. In cases that the seeker has some concerns on the suggested site or time and date, he can comment on the event or decline the event. The facilitator agrees to the terms and conditions of the application. They are not allowed to divulge to anyone any information about the seekers in the application. The system administrator can revoke the use of the application to any facilitator who fails to comply with the agreement.

Figure 6 shows the recommended group activities for a specific event based on the group's problems and interests. The group should perform these activities actually, not virtually. After the gathering, the group can assess whether the group session has helped them cope with their depression or current struggles.

And lastly, Figure 7 shows sample a discussion after an event. Here, the seeker can express more about his experiences at the gathering which will serve as an avenue for the facilitator to ascertain whether the activity has helped the seeker or not. 


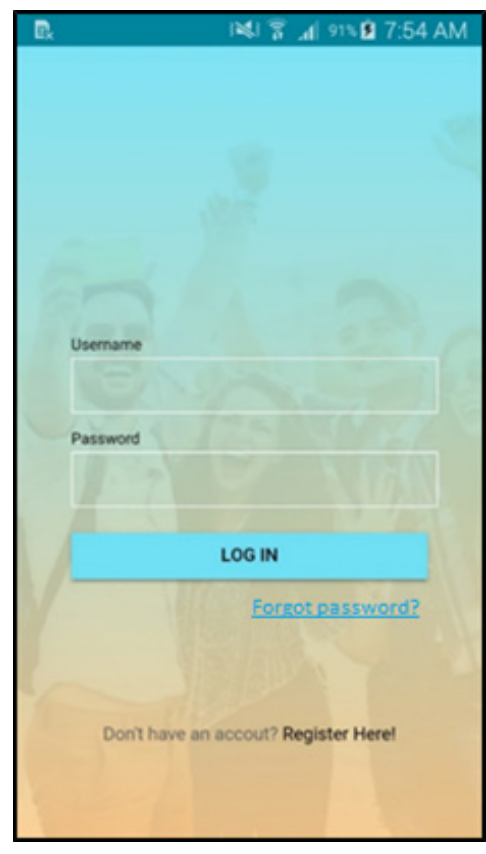

Figure 1. Login Page

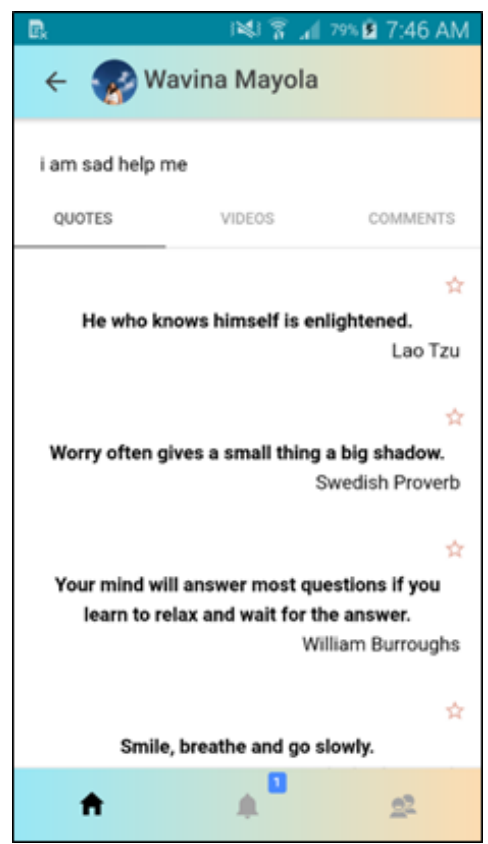

Figure 2. Quotes on the Media Wall

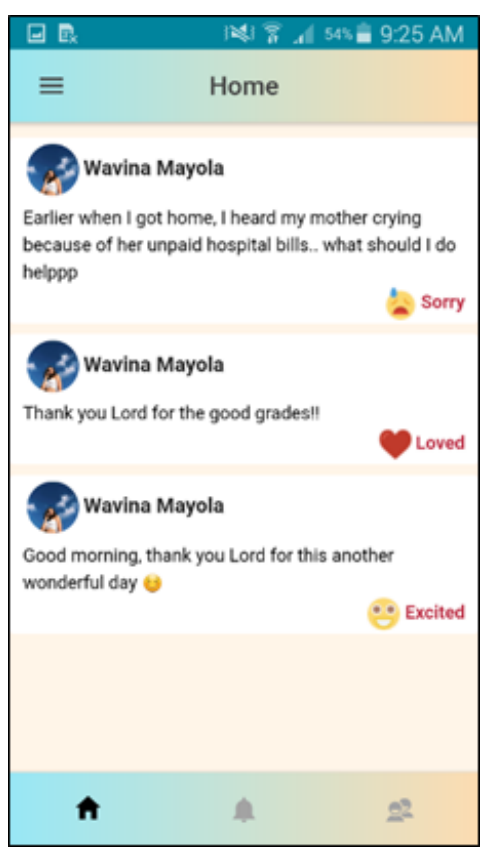

Figure 3. Freedom Wall

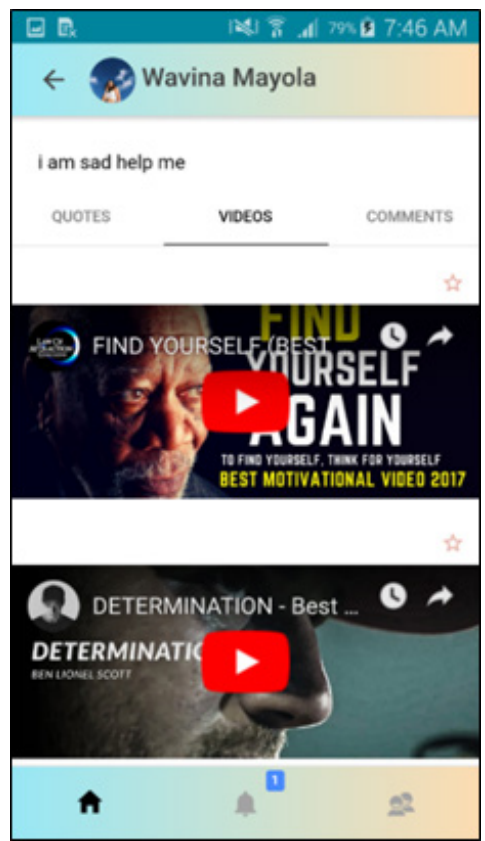

Figure 4. Video Media Wall 


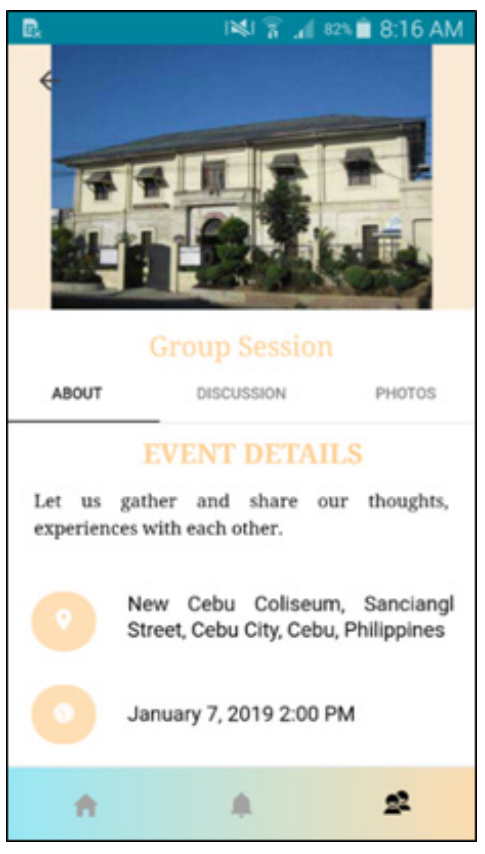

Figure 5. Event Details

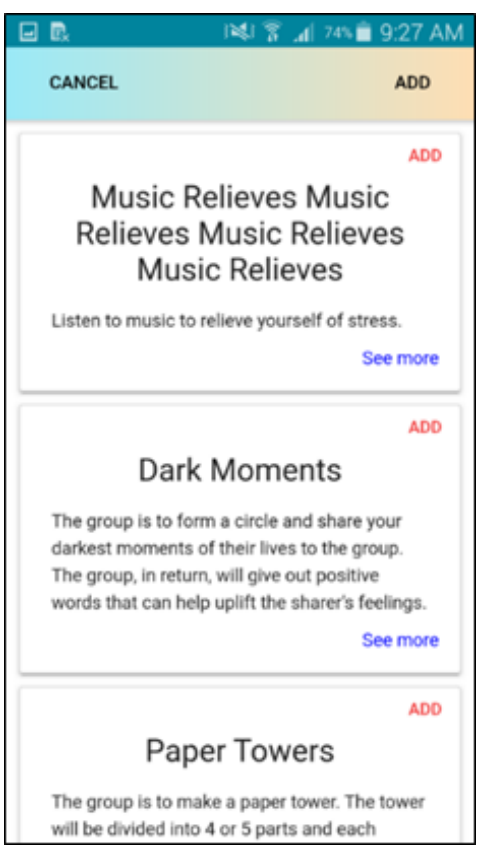

Figure 6. Recommended Activities

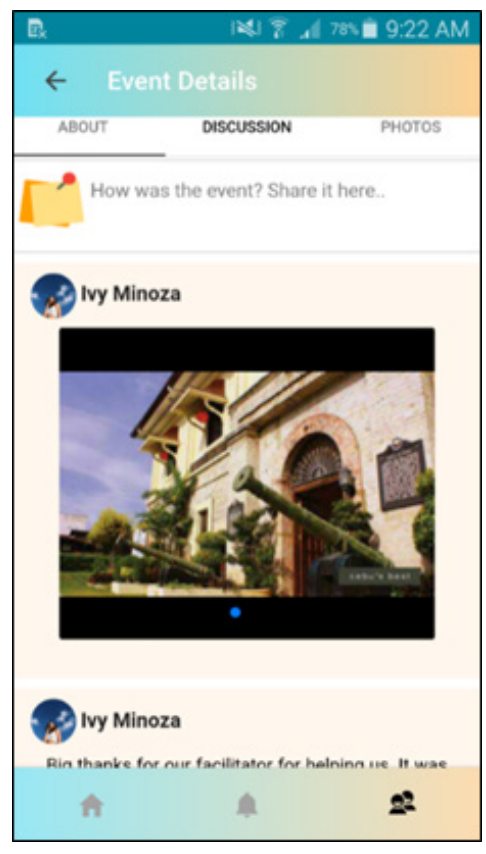

Figure 7. Event Discussion

\section{Performance Testing}

This section shows the computation of the responsiveness and stability of the application. It includes retrieving and storing data from the server to the mobile application and vice versa. The main functionalities are the only features tested in this section. Table 7 displays the average result after running each test case four times.

Table 7. Performance Testing

\begin{tabular}{lc}
\hline Test Case & $\begin{array}{c}\text { Average Running } \\
\text { Time }\end{array}$ \\
\hline Generate Talk Circle & 11.3675 Seconds \\
$\begin{array}{l}\text { Generate Recommended } \\
\text { Activities }\end{array}$ & 5.6525 Seconds \\
$\begin{array}{l}\text { Generate Recommended } \\
\text { Venues }\end{array}$ & 3.065 Seconds \\
\hline
\end{tabular}


Based on Table 7, generating a talk circle takes the longest time among other activities. The number of times at which the sorting and mapping processes of each seeker and facilitator is cued is the reason behind the length of the process. Another observation is that the recommended activities take more time than the process of recommending the venues. The cause of this delay is the additional sorting process, before matching to the group's problems and interest. It would take time than comparing the coordinates in the recommendation of venues. The computational cost is directly proportional to the number of data sets. Since this study uses KNN, this situation is inevitable.

\section{Accuracy Testing}

This testing phase shows two confusion matrices. K-fold cross-validation is another validation technique. However, this study uses the confusion matrix (Confusion Matrix in Machine Learning, n.d.), which is widely accepted. The researchers cross-check the prediction of the algorithm with human experts' classification. The accuracy of KNN is determined through the predicted result of the algorithm vs. the human experts' classification. The actual classification done by the human experts is in the second and third rows of Table 8 and Table 9. The second and third columns of Table 8 and Table 9 shows the predicted classification produced by the algorithm.

Table 8 displays the result after conducting a test whether the seeker belongs to the right talk circle or not. The algorithm has two predicted classifications, correct talk circle and incorrect talk circle. If the system correctly matches the seeker's problem with the facilitator's specialization, the seeker belongs to the correct talk circle. Otherwise, the researcher adds the seeker to the incorrect talk circle. There are 1000 seekers in the dataset, and the algorithm predicted 650 seekers who belong correctly to the talk circle, and incorrectly matched the 350 of them. In reality, there are 675 seekers who belong correctly to the talk circle, and 325 of them do not belong to the correct talk circle. With these figures, the study uses Equation 4 to get the accuracy of the algorithm. This equation yields an $86 \%$ accuracy rate.

Table 8. Accuracy Testing for Talk Circle

\begin{tabular}{lccc}
\hline & $\begin{array}{c}\text { Correct } \\
\text { Talk } \\
\text { Circle }\end{array}$ & $\begin{array}{c}\text { Incorrect } \\
\text { Talk } \\
\text { Circle }\end{array}$ & $\begin{array}{c}\text { Actual } \\
\text { Total } \\
\text { Count }\end{array}$ \\
\hline $\begin{array}{l}\text { Correct Talk } \\
\text { Circle }\end{array}$ & 592 & 83 & 675 \\
$\begin{array}{l}\text { Incorrect Talk } \\
\text { Circle }\end{array}$ & 58 & 267 & 325 \\
$\begin{array}{l}\text { Predicted } \\
\text { Total Count }\end{array}$ & 650 & 350 & 1000 \\
\hline
\end{tabular}

Table 9 shows the result of the use of algorithm for classifying whether or not a set of activities for a particular talk circle is significant in helping the seekers cope with their problems. Out of the 500 sets of activities in the database, 333 of them are predicted by the algorithm to be significant, and 167 are insignificant. Although actually, there are 337 relevant sets of activities, and 163 are arbitrarily selected. With this result, the algorithm yields an $82 \%$ accuracy rate using Equation 4.

Equation 4: Average Accuracy

$$
\text { Accuracy }=\frac{T P+T N}{T P+T N+F P+F N}
$$


The study uses Equation 4 (Confusion Matrix in Machine Learning, n.d.) for the accuracy test of the confusion matrices. True Positive (TP) is the value that the algorithm correctly predicted as actual positive; true negative (TN) is a correct negative value; false positive (FP) is the incorrect positive value; and false negative (FN) is a positive value predicted as negative. The algorithm yields an $84 \%$ accuracy rate after getting the average of both results. In KNN, if $\mathrm{k}$ is too small, it is sensitive to noise or misclassified data. If $\mathrm{k}$ is too large, the classification may include points from other groups. Thus, evaluating more on the best $k$ for grouping the seekers, and the attributes of the significant activities should be noted to improve the accuracy rate of the system.

Table 9. Accuracy Testing for Recommended Activities

\begin{tabular}{lccc}
\hline & $\begin{array}{c}\text { Significant } \\
\text { Activities }\end{array}$ & $\begin{array}{c}\text { Insig- } \\
\text { nificant } \\
\text { Activities }\end{array}$ & $\begin{array}{c}\text { Actual } \\
\text { Total } \\
\text { Count }\end{array}$ \\
\hline $\begin{array}{l}\text { Significant } \\
\text { Activities }\end{array}$ & 290 & 47 & 337 \\
$\begin{array}{l}\text { Insignificant } \\
\text { Activities }\end{array}$ & 43 & 120 & 163 \\
$\begin{array}{l}\text { Predicted } \\
\text { Total Count }\end{array}$ & 333 & 167 & 500 \\
\hline
\end{tabular}

\subsection{Conclusion}

The lazy supervised algorithm in this study can still be improved. It has an accuracy rate of $84 \%$, combining the accuracy testing results of the generated talk circle and recommended physical activities. This study deals with individuals who are seeking help from reliable and professional counselors. Thus, this study recommends that the margin of error be lessened if removing the error is inevitable. The researchers commend the exploration of which $\mathrm{K}$ gives the minimum validation error. The performance testing of the app, especially in generating the talk circle, is greatly affected by the computation time of KNN. Since KNN suffers the redoing of the classification process, speeding up the process by integrating KD Tree or Ball Tree might help solve the prediction cost. However, the researchers believe that the application still serves its purpose. Those who are feeling lonely and isolated can always use the application to post their feelings and be counseled by a professional counselor or psychiatrist. They can read inspirational quotes and watch motivational videos on their walls. They can also have social support groups with whom they can feel a sense of belonging and acceptance. With the set of activities recommend to the group, the seekers can enjoy the company with other seekers and unwind themselves with the idyllic scenery of the venue. Indeed, loneliness and social isolation are both subjective and objective. There are numerous solutions to lessen their adverse effects. With this application, the researchers hope to promote healthy well-being for lonely and socially isolated individuals.

\section{References}

Aha, D. W. (1997). Lazy Learning. Springer, Dordrecht.

Andrade, A. (2016). K Nearest Neighbor and Lazy Learning. Retrieved June 25, 2020, from Data Science Guide: https://datascienceguide. github.io/k-nearest-neighbor 
Cattan, M., White, M., Bond, J., \& Learmouth, A. (2005). Preventing social isolation and loneliness among older people: a systematic review of health promotion interventions. Ageing \& Society, 25(1), 41-67.

Chen, C.-C., Petrick, J., \& Shahvali, M. (2016). Tourism Experiences as a Stress Reliever: Examining the Effects of Tourism Recovery Experiences on Life Satisfaction. Journal of Travel Research, 55(2), 150-160.

Cherry, K. (2018, Nov 16). Social Support Is Imperative for Health and Well-Being. Retrieved Jan 15, 2020, from Verywell Mind: https://www. verywellmind.com/social-support-forpsychological-health-4119970.

Cobb, S. (1976). Social Support as a Moderator of Life Stress. Psychosomatic medicine, 38(5), 300-314

Cohen, S., \& Wills, T. (1985). Stress, Social Support, and the Buffering Hypothesis. Psychological Bulletin, 98(2), 310-357.

Confusion Matrix in Machine Learning. (n.d.). Retrieved Jan 15, 2020, from GeeksforGeeks: https://www.geeksforgeeks.org/confusionmatrix-machine-learning/

Cutrona, C., \& Russell, D. (1993). The provisions of social relationships and adaptation to stress. American Journal of Orthopsychiatry, 63(4), 597-605.

Equation of a Circle. (2007). Retrieved Jan 15, 2020, from Varsity Tutors: https://www. varsitytutors.com/hotmath/hotmath_help/ topics/equation-of-a-circle

Euclidean Distance. (n.d.). (NIST, Producer) Retrieved Jan 15, 2020, from Statistical Engineering Division: https://www.itl.nist. gov/div898/software/dataplot/refman2/ auxillar/eucldist.htm

Garlow, S., Rosenberg, J., Moore, D., Haas, A., Koestner, B., Hendin, H., \& Nemeroff, C. (2008). Depression, desperation, and suicidal ideation in college students: results from the American Foundation for Suicide Prevention College Screening Project at Emory University. Depression and anxiety, 25(6), 482-488.

Gazda, G. (1969). Group Counseling: A Development Approach. Canadian Journal of Counselling and Psychotherapy/Revue canadienne de counseling et de psychothérapie, 3(4).

Geolocation API. (n.d.). Retrieved Jan 15, 2020, from https://developers.google.com/maps/ documentation/geolocation/intro

Grippo, A., Gerena, D., Huang, J., Kumar, N., Shah, M., Ughreja, R., \& Carter, C. (2007). Social isolation induces behavioral and neuroendocrine disturbances relevant to depression in female and male prairie voles. Psychoneuroendocrinology, 32(8-10), 966980.

Happify: Science-Based Activities and Games. (n.d.). Retrieved Jan 15, 2020, from https:// www.happify.com/ 
Kataria, A., \& Singh, M. D. (2013). A Review of Data Classification Using K-Nearest Neighbour. International Journal of Emerging Technology and Advanced Engineering, 3(6).

Maps JavaScript API. (n.d.). Retrieved Jan 15, 2020, from https://developers.google.com/maps/ documentation/javascript/tutorial

Movable Type Scripts. (n.d.). Retrieved Jan 15, 2020, from Calculate distance, bearing and more between Latitude/Longitude points: https:// www.movable-type.co.uk/scripts/latlong. html

Quotes and expressions. The most inspiring expressions of mankind. (n.d.). Retrieved Jan 15, 2020, from https://forismatic.com/en/

Satapathy, S., \& Naik, A. (2016). Social group optimization (SGO): a new population evolutionary optimization technique. Complex \& Intelligent Systems, 2(3), 173-203.

Stigma: Mood Tracker and Journal. (n.d.). Retrieved Jan 15, 2020, from https://psyberguide.org/ apps/stigma/

Stults-Kolehmainen, M. A., \& Sinha, R. (2014). The effects of stress on physical activity and exercise. Sports medicine, 44(1), 81-121.

Talkspace. (n.d.). Retrieved Jan 15, 2020, from Talkspace: shorturl.at/aqL27

Tomaka, J., Thompson, S., \& Palacios, R. (2006). The relation of social isolation, loneliness, and social support to disease outcomes among the elderly. Journal of aging and health, 18(3), 359-384.

Truschel, J.(2020). Top 25 Mental Health Apps for 2018: An effective alternative for when you can't afford theraphy? Retrieved from https:// www.psycom.net/25-best-mental-healthapps

University of California at San Francisco PRIME. (2016). Retrieved from https://apps.apple. com/tr/app/ucsf-prime/id1031402495

Wenger, G., Davies, R., Shahtahmasebi, S., \& Scott, A. (1996). Social isolation and loneliness in old age: review and model refinement. Ageing \& Society, 16(3), 333-358.

Youtube API. (n.d.). Retrieved Jan 15, 2020, from https://developers.google.com/youtube/v3 